\title{
The impact of post acquisition period on detection of Candidatus Liberibacter solanacearum in tomato potato psyllid
}

\author{
W.R.M. Sandanayaka ${ }^{1}$, L.K. Tooman ${ }^{1}$ and R.E. Hewett ${ }^{2}$ \\ ${ }^{1}$ The New Zealand Institute for Plant E Food Research Limited, Private Bag 92-169, \\ Auckland 1142, New Zealand \\ ${ }^{2}$ Victoria University of Wellington, P O Box 600, Wellington 6140, New Zealand \\ Corresponding author: Manoharie.Sandanayaka@plantandfood.co.nz
}

\begin{abstract}
The impact of post acquisition period (PAP) on detection of Candidatus Liberibacter solanacearum (Lso) in adult tomato potato psyllid (TPP; Bactericera cockerelli) was investigated using qPCR assays on whole insects. Lso in adults was initially tested immediately after acquisition access periods (AAP) of 15, 24, 48, 72, $96 \mathrm{~h}$ or 1 week on Lsoinfected tomato leaves. These results were compared with those from adults with 15 or $24 \mathrm{~h}$ AAP followed by 24-, 48- or 72-h PAP on Lso-free tomato leaves. From 15-20 individuals of each treatment tested, 5-15\% were positive for Lso immediately after AAP of 15 or 24 h. That increased to $84 \%$ and $100 \%$ with $24-$ and 48 -h PAPs respectively, but decreased to 25-47\% after 72 h. Longer AAP ( 48 h-1 week) without PAP did not exceed 55\% acquisition success. The phloem ingestion and possible acquisition of Lso during 15 and $24 \mathrm{~h}$ plant access periods are discussed.
\end{abstract}

Keywords tomato/potato psyllid, Candidatus Liberibacter solanacearum, post acquisition period, phloem ingestion, detection.

\section{INTRODUCTION}

Zebra chip is an economically important disease of potato (Solanum tuberosum L.) in the United States, Mexico, Central America and New Zealand (Munyaneza et al. 2007; Munyaneza 2012). This disease is caused by Candidatus Liberibacter solanacearum (Lso), also known as Candidatus Liberibacter psyllaurous (Hansen et al. 2008). Lso has been shown to severely disrupt carbohydrate flow in potato plants, leading to Zebra chip symptoms in the tubers (Buchman et al. 2011). Lso is a phloem-limited, Gram-negative, unculturable bacteria that was initially found in solanaceous crops (Liefting et al. 2008, 2009) and is transmitted by tomato potato psyllid (TPP) Bactericera cockerelli (Šulc) (Hemiptera: Triozidae) (Munyaneza et al. 2007; Hansen et al. 2008). This bacterium was found later in carrot plants in Finland, Sweden and Norway, where it was vectored by Trioza apicalis Forster (Munyaneza 2012). Recent reports from the Canary Islands and Spain have found Lso in carrot and celery crops where it is vectored by B. trigonica (Alfaro-Fernandez et al. $2012 \mathrm{a}, \mathrm{b}$ ).

TPP was first reported in New Zealand in 2006 and has now established throughout the North and South Island. Lso disease symptoms 
were first found in tomato crops in New Zealand in 2008 (Liefting et al. 2009), and the distribution of Lso in the country matched the known general distribution of its vector TPP (Teulon et al. 2009). Ogden (2011) reported that the economic loss to the potato industry in New Zealand caused by the incursion of TPP and Lso was at least \$120 million, including \$28 million in the 2010-11 growing season alone.

TPP is a phloem feeder, with an extensive host range on plant species within the families Solanaceae, Convolvulaceae and Laminaceae (Martin 2008). It has been reported that TPP feeding behaviour is important in the transmission of Lso into host plants (Buchman et al. 2011; Levy et al. 2011). The electrical penetration graph (EPG) technique, which electrically records stylet penetration by Hemipterans (Tjallingii 1978), has been used to monitor the feeding behaviour of adult TPP (Sanadanayaka et al. 2011, 2012; Butler et al. 2012).

Previous studies have reported that acquisition of Lso by TPP occurred during $24 \mathrm{~h}$ acquisition access periods (AAP) (Rashed et al. 2012). These authors also reported that acquisition success was influenced by the feeding site on the plant with the highest acquisition success (ca 52\%) occurring when insects had access to the whole plant. Very little is known about the retention of Lso inside the TPP body. Inoue et al. (2009) suggested that multiplication of the bacterium in the psyllid is essential for efficient transmission. The location of a related bacterium, Candidatus Liberibacter asiaticus (Las), within the body of its psyllid vector, the Asian citrus psyllid Diaphorina citri, has been described by Ammar et al. (2011). Using quantitative polymerase chain reaction (qPCR), scanning electron microscopy and fluorescence in situ hybridization techniques, they confirmed the presence of Las in salivary glands, haemolymph, alimentary canal, Malpighian tubules, filter chamber, midgut, fat, muscle and ovaries. Their findings indicated a systemic presence of the bacteria within $D$. citri and suggested that the pathogen spreads to the organs within 1 to 2 days of acquisition.
The primary aim of this study was to test the effect of different post acquisition periods on the detection of Lso in TPP using qPCR, based on the assumption that Lso multiplies in TPP after acquisition. The efficiency of feeding of TPP during $15 \mathrm{~h}$ and $24 \mathrm{~h}$ plant access periods was also measured.

\section{MATERIALS AND METHODS Insects and plants}

Lso-positive and -negative TPP colonies were maintained in separate glasshouses. The Lsonegative TPP colony was reared on clean (Lsofree) capsicum plants (Capsicum annuum L. 'Giant Bell') and the Lso-positive TPP colony was reared on tomato plants (Solanum lycopersicum L. 'Moneymaker') showing Lso symptoms and testing positive for Lso in regular qPCR tests. Both colonies were maintained in $50 \times$ $50 \times 50 \mathrm{~cm}$ steel frame cages covered with fine net at $24 \pm 1^{\circ} \mathrm{C}$ and under natural light. Insect samples from both colonies were tested monthly by qPCR to confirm presence or absence of Lso. Adults from the Lso-negative TPP colony were used in the experiments. Tomato plants ('Moneymaker') were infected with Lso by exposing groups of six clean plants to 50-60 adult TPP from the Lsopositive colony for a week in a $50 \times 50 \times 70 \mathrm{~cm}$ steel frame cage covered with fine net. Those plants were then sprayed with Confidor ${ }^{\otimes}$ at $1 \mathrm{~g} /$ litre to kill all the psyllids and left in a separate room in the glasshouse until symptoms typical of Lso appeared. The presence of Lso in symptomatic plants was confirmed by qPCR tests. Lso-free tomato plants ('Moneymaker') were grown from seeds in individual pots $(10 \times 10 \times 11.5 \mathrm{~cm})$ with potting mix in an insect- and pathogen-free glasshouse.

\section{Acquisition access periods}

In the first experiment, TPP adults from the Lsofree colony were exposed to excised symptomatic leaves of Lso-positive tomato plants for AAPs of $15,24,48,72$ or 96 h or 1 week. Each excised leaf from a Lso-infected tomato plant was placed into a vertically-held Petri dish $(85 \times 15 \mathrm{~mm})$, with the stem extending through a foam plug fitted into an opening cut in the base of both 
the lid and the dish. The end of the stem was submerged in a $50 \times 10 \mathrm{~mm}$ tube of water held under the Petri dish and the tube was plugged with the foam. Twenty TPP were left on each leaf for each of the assigned AAPs (a total of 120 TPP on six excised leaves) after which the insects were individually collected into $0.6 \mathrm{ml}$ tubes and stored dry at $-20^{\circ} \mathrm{C}$ for subsequent qPCR testing for Lso. Two samples from each leaf were also collected for qPCR tests to confirm the presence of Lso in the leaves. A logistic regression model was used to test the relationship between AAP and the number of Lso-positive TPP. The model was fitted using the GENMOD procedure in SAS, allowing for over-dispersion of the binomial (SAS Institute Inc. 2008).

\section{Post-acquisition period}

The impact of three different post-acquisition periods (PAPs) on detecting Lso in TPP was tested in the second experiment. Twenty TPP adults were placed on each of six Lso-symptomatic leaves excised from Lso-positive tomato plants, set up as described for the first experiment. Sixty TPP were removed after a 15 -h AAP and immediately released onto a Lso-free tomato plant housed in a cage $(50 \times 50 \times 50 \mathrm{~cm})$ covered with fine net. The remaining sixty TPP on Lsopositive leaves were removed after a 24-h AAP and immediately released onto another Lso-free tomato plant in a cage. Groups of 15-20 TPP were then removed from each plant after 24, 48 and $72 \mathrm{~h}$ of PAPs and individually stored in $0.6 \mathrm{ml}$ tubes at $-20^{\circ} \mathrm{C}$ for subsequent qPCR testing for Lso.

Both experiments were carried out at $24 \pm 1^{\circ} \mathrm{C}$ and 16:8 h (light:dark) periods.

\section{EPG monitoring to measure real feeding time}

The duration of actual phloem ingestion by tethered TPP adults was measured for $15-\mathrm{h}$ and 24-h plant access periods in a follow-up EPG test. The aim of this test was to estimate the actual periods TPP spent ingesting phloem and potentially acquiring Lso from infected leaves during the AAPs tested in the second experiment. The stylet penetration activities of $20 \mathrm{TPP}$ adults
(Lso-free) on shoots of Lso-infected tomato plants were recorded for $24 \mathrm{~h}$. To prepare for the EPG tests, adults were collected from the Lso-free colony into $1.5 \mathrm{ml}$ tubes (4-5 adults/tube) and the insects were immobilised under $\mathrm{CO}_{2}$ for $2-3 \mathrm{~s}$ before wiring. Individual TPP were attached to a $2 \mathrm{~cm}$ long, $18.5 \mu \mathrm{m}$ diameter gold wire, with a drop of silver conductive paint ( $n$-butyl acetate solvent; Ladd Research Industries, Williston, VT). The other end of the gold wire was attached to a $3 \mathrm{~cm}$ long copper wire connected to the head of a $3 \mathrm{~mm}$ diameter copper nail, which fitted tightly into the input probe of the EPG monitor. Wired insects were left for $15 \mathrm{~min}$ to recover before being placed on the abaxial surface of Lso-infected tomato leaves. The plant electrode (a thick copper wire) was inserted into the waterfilled tube into which the leaf stem had been placed. The stylet penetration of each insect was monitored for $24 \mathrm{~h}$ on a Giga-4 DC-EPG system (WF Tjallingii, Wageningen, The Netherlands) under fluorescent lights for a $16 \mathrm{~h}$ photoperiod with the photo phase of $6 \mathrm{am}-10 \mathrm{pm}$ at $24 \pm 1^{\circ} \mathrm{C}$. Four insects were monitored on four channels of the EPG monitor simultaneously, and a total of 20 insects was monitored. Data output was digitized using DI-720 analogue to digital board (DATAQ $^{\circledR}$ instruments). Stylet penetration data were acquired, stored and measured using WinDaq Pro+ software (DATAQ ${ }^{\circledast}$ instruments). The duration between the start of the recording and the first phloem ingestion of individual TPP was measured. The total phloem ingestion period of each TPP was also measured for the first $15 \mathrm{~h}$ of the recording and then the full recording period $(24 \mathrm{~h})$. The efficiency of feeding by individual TPP (calculated by dividing the total duration of phloem ingestion by the recording period) for the first $15 \mathrm{~h}$ and the last $9 \mathrm{~h}$ of the total $24 \mathrm{~h}$ recording period were compared using a paired-t test of the null hypothesis that the average of the differences between two efficiencies is 0 .

\section{Detection of Lso}

Individual plant samples were prepared for testing by grinding with a Qiagen Tissue Lyser and the DNA extracted with a QIAxtractor ${ }^{\circledR}$ 
system according to the manufacturer's protocol (QIAgen, 2010). Individual TPP were processed according to the Zygem protocol outlined in Beard \& Scott (2013). The qPCR analysis to detect Lso was performed according to the method of Beard \& Scott (2013), with a modification being a transfer to the Roche 480 LightCycler $^{\circledR}$ Platform (Roche Diagnostics) using Roche Sybr Green I Master Mix. This detection platform has a larger sample number capacity, allowing both the Lso-exposed and control samples to be run in unison. An observed Ct shift meant samples were run for 45 cycles instead of 40 (see Puketapu et al. (2013) for a more detailed description of detection methods). Samples that amplify in the last five cycles of a qPCR programme are at the limits of detection for this test and detection standards ranged from $1 \times 10^{9}$ gene copies to 10 copies. However, only $2 \mu$ l of the total $50 \mu$ DNA extraction of each psyllid was used, therefore, it was possible that positive individuals with a very low Lso titre would not be detected before cycle 40 . It was therefore decided that in the case where one or more of the three replicates tested positive after cycle 40 and the product showed the correct melt curve, the sample was counted as positive when interpreting the data.

\section{RESULTS}

All the leaves used for Lso acquisition were confirmed to be positive for Lso by qPCR. Results revealed that a single TPP can acquire Lso within $15 \mathrm{~h}$ of being placed on an infected plant, but not more than $15 \%$ of the insects were detected as positive for Lso when tested immediately after a 15- or 24-h AAP (Figure 1). The proportion of Lso-positive insects increased to $55 \%$ when insects were tested after a 48 -h AAP or longer. The results of the logistic model showed evidence of a relationship ( $\mathrm{P}=0.0377)$ between the percentage TPP testing positive for Lso and the length of the AAP. However, the fitted curve showed a trend of increasing numbers of Lso-positive TPP with increasing AAP.

The PAP on Lso-free tomato plants increased the proportion of Lso-positive TPP in groups with 15- or 24-h AAP, compared with those without PAP (Figure 2). All TPP with a 15-h AAP followed by a 48-h PAP were found to be positive for Lso. TPP that had a 24-h AAP followed by a 24-h PAP were $84.2 \%$ positive for Lso. The proportion of TPP with Lso was less in TPP groups with a 72-h PAP than the groups with 24-h or 48-h PAP.

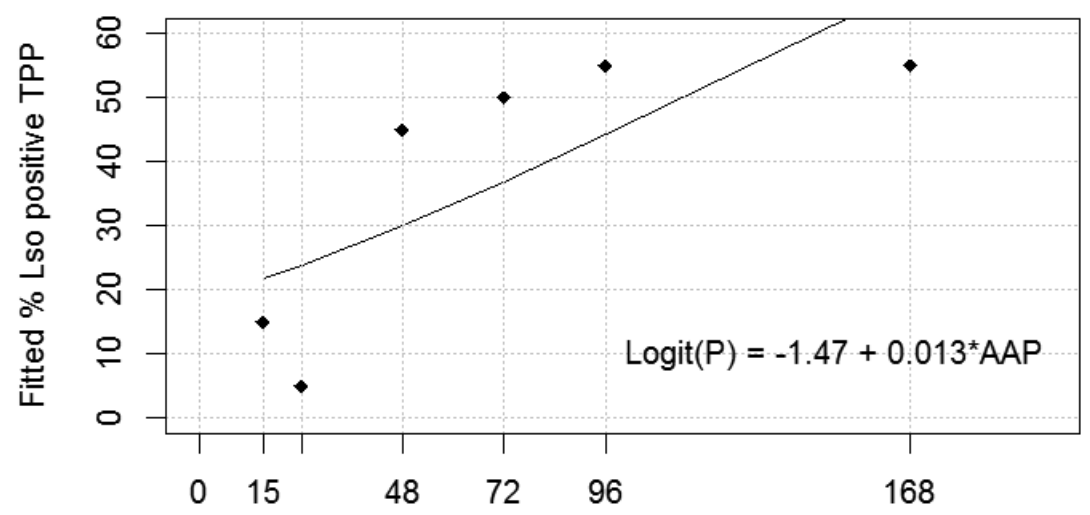

Acquisition access period (h)

Figure 1 The observed values and fitted line showing the relationship between the percentage tomato potato psyllids testing positive by qPCR for Candidatus Liberibacter solanacearum (Lso) immediately after exposure to Lso-infected plants and the time spent on the plants (acquisition access period). 


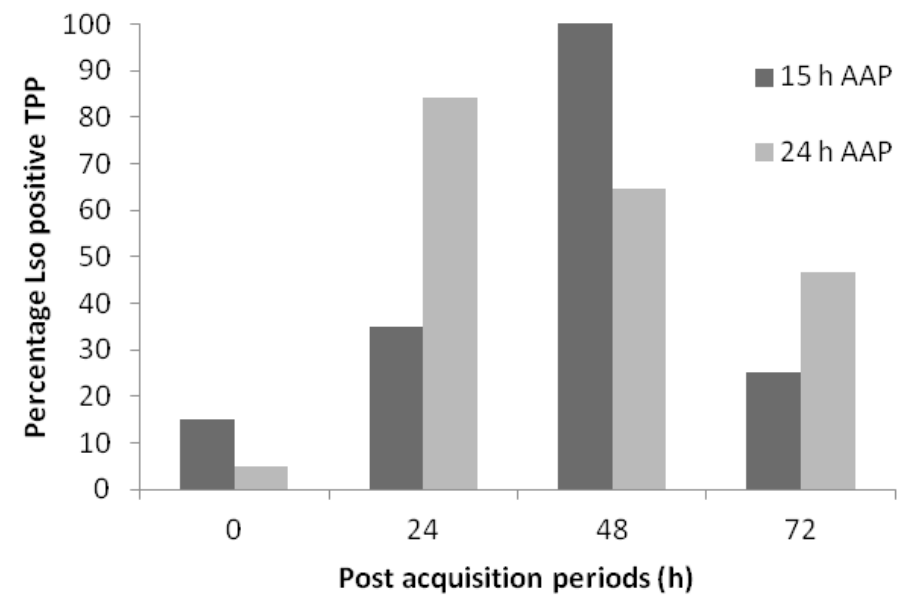

Figure 2 The percentage of tomato potato psyllids testing positive for Candidatus Liberibacter solanacearum (Lso) by qPCR after 15 or $24 \mathrm{~h}$ of exposure to Lsoinfected plants (acquisition access period, AAP) followed by $0,24,48$ or $72 \mathrm{~h}$ of post acquisition periods on healthy plants.

The phloem ingestion periods of tethered TPP measured in the follow-up EPG test provided an estimate of the duration of feeding of the TPP given 15-h and 24-h AAPs in the other experiments. The EPG waveforms were characterised as shown in Butler et al. (2012). The recordings started with non-probing base line (np) (Figure 3 ). The initial electrical contact of stylet with plant tissues (probe) appeared with the highest amplitude waveform, which continued with a mixture of waveforms with different frequencies, representing saliva secretion while probing in the vicinity of epidermal and parenchyma cells (C waveform) and sometime xylem ingestion ( $G$ waveform). The initial contact with phloem tissues was indicated by $\mathrm{D}$ waveform, which was usually followed by phloem salivation (E1 waveform) and then phloem ingestion (E2 waveform). E2 frequently changed to E1 during the phloem phase. Two of 20 TPP monitored

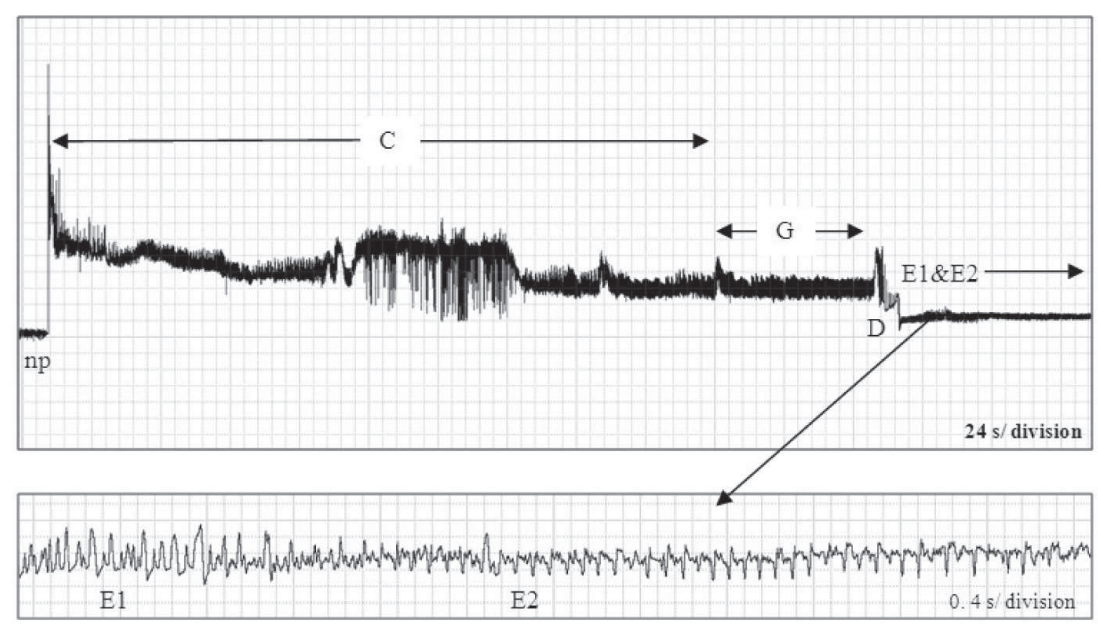

Figure 3 An overview of electrical penetration graph recordings of the stylet penetration behaviour of tomato potato psyllids on tomato leaves. The upper plot shows non-probing baseline (NP), saliva secretion while probing in the vicinity of epidermal and parenchyma cells $(C)$, ingestion from xylem tissues $(G)$ and initial contact with phloem tissues (D). Expanded phloem salivation (E1) waveforms and phloem ingestion (E2) waveforms are shown in the lower plot. 


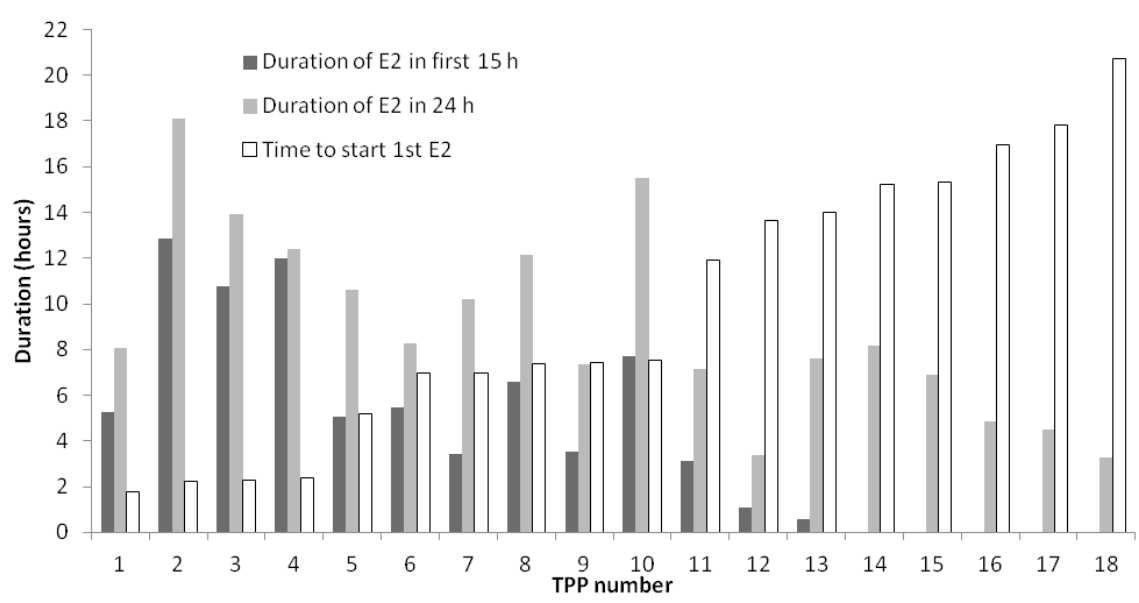

Figure 4 Real time feeding (phloem ingestion) of 18 tomato potato psyllids during the first $15 \mathrm{~h}$ and full electrical penetration graph recording period of $24 \mathrm{~h}$.

on EPG did not show phloem ingestion during the $24 \mathrm{~h}$ of recording so they were not included in the graph (Figure 4). Thirteen TPP started ingesting phloem during the first $15 \mathrm{~h}$ and 18 TPP showed phloem ingestion during $24 \mathrm{~h}$. Variability in phloem ingestion was noticed between individual TPP. The mean duration between the start of the recordings and the first phloem ingestion was $11.19 \pm 1.6 \mathrm{~h}$, with a minimum duration of $107 \mathrm{~min}$. The mean duration of phloem ingestion measured within the first $15 \mathrm{~h}$ and $24 \mathrm{~h}$ of recordings was $5.96 \pm 1.08$ and $9.01 \pm 1.05 \mathrm{~h}$ respectively. T-test results indicated that the average efficiency of feeding in the last $9 \mathrm{~h}$ was significantly higher $(\mathrm{P}=0.031)$ than that in the first $15 \mathrm{~h}$.

\section{DISCUSSION}

The results demonstrated a trend of detectable Lso infections in TPP increasing with longer AAPs. It is suggested that PAPs had an impact on qPCR results as more psyllids tested positive for Lso after a PAP than when tested immediately after their AAP. Variability in qPCR results from TPP tested after acquisition may also be influenced by the Lso titre at the acquisition site and the length of time required for accumulation or multiplication of Lso in insect bodies. It has been shown previously that Lso acquisition occurs during phloem ingestion on an infected plant (Sandanayaka et al. 2012).

In contrast to Beard et al. (2013), samples returning only $1 / 3$ positives were considered to be positive in the present analysis, instead of $2 / 3$. This is because the original method of Beard et al. (2013) had a detection limit of 5 bacterial genome copies, but transfer to the Roche 480 Light Cycler platform meant that the limit of detection was raised to 10 bacterial genome copies per extraction. Thus, a positive on the Roche 480 Light Cycler platform meant that a much greater number of copies of the bacterial genome was present. It is recognised that PCR runs above 40 cycles can produce artefacts or non target amplifications giving false positives. However, in the present study the addition of a melt curve assessment of the $\mathrm{qPCR}$ product was used to confirm that the right target was being amplified. As a single bacterium can multiply to a larger, more readily detectable, titre given enough time, it was felt that the $1 / 3$ positive approach allowed for dilution factor effects associated with the large DNA extraction volume.

EPG recordings revealed that $65 \%$ and $90 \%$ of tethered TPP ingested from phloem tissues during plant access periods of $15 \mathrm{~h}$ and $24 \mathrm{~h}$ respectively. It can be assumed that the free TPP in the first and 
the second experiments were as likely to feed on phloem during the same plant access periods $(15 \mathrm{~h}$ and $24 \mathrm{~h}$ ) as the tethered insects. Thus, it is likely that the proportions of free TPP that acquired Lso in AAPs of 15 or $24 \mathrm{~h}$ would be equal to or greater than those observed in the EPG experiment. The average time taken by a tethered TPP to start phloem ingestion from the beginning of the plant access period was $11.19 \mathrm{~h}$. All of the TPP with $15-\mathrm{h}$ AAP followed by a 48-h PAP tested positive for Lso, indicating that all TPP had acquired Lso, which suggested that the combination of PAP and AAP improved detection of Lso in TPP. The results of qPCR after a $24-\mathrm{h}$ PAP showed that $84.2 \%$ of TPP acquired Lso during a 24-h AAP, which correlates to EPG data showing $90 \%$ of TPP successfully ingested phloem during a 24-h plant access period. Therefore it is suggested that every single TPP that succeeded in feeding and acquiring Lso after 15- or 24-h AAPs in the first experiment may not have detected as positive for Lso, when tested immediately after AAP. Presumably the success of detection may be impacted by the amount of Lso acquired by the TPP during the AAP and the length of time available for Lso to multiply inside the insect body during any PAP.

Tethered TPP demonstrated that the efficiency of feeding in the first $15 \mathrm{~h}$ is lower than that in the last $9 \mathrm{~h}$ as the insects may need some adaptation time to a new environment. Although the tethering effect on the insect behaviour has to be considered (Tjallingii 1986), an average TPP spent a considerable proportion of the first $15 \mathrm{~h}$ attempting to locate phloem tissues and spent more time feeding during the last $9 \mathrm{~h}$. When phloem tissues were located by the TPP, most of the time was spent on phloem ingestion and most likely acquiring Lso. Therefore it is suggested that a greater number of TPP acquire Lso when given a 24-h AAP than with a 15-h AAP.

The proportions of Lso-positive TPP decreased when TPP had a 72-h PAP, suggesting that longer feeding on Lso-free plants may reduce the Lso concentration in the TPP's body. This agrees with the findings of Pelz-Stelinski et al. (2010) who reported that the proportion of Las-positive adult D. citri, determined using $\mathrm{qPCR}$, decreased over time when held on healthy plants. Declines in bacteria titre in the insect over time may be the result of initial concentrations of bacteria acquired by the insect, age of the insect or negative effects of the bacterium on the insect host (Purcell 1982).

In the first and the second experiments, Lso donor leaves were picked from the same tomato plant to reduce the possible influence of the physiology of host plant on acquisition. A small number $(n=20)$ of TPP were tested on each Lso donor leaf to avoid competition for feeding, especially during longer plant access periods. Although an increased acquisition rate was expected from TPP after the longer confinement on Lso-infected leaves, the number of TPP that tested positive for Lso changed little among the TPP groups that had $48 \mathrm{~h}, 72 \mathrm{~h}, 96 \mathrm{~h}$ or 7 days of AAP. Pelz-Stelinski et al. (2010) suggested that longer confinement of $D$. citri on Las-infected plants may allow time for the bacterial titre to increase in the insect. In the present study excised leaves from an infected plant were used as the source of Lso for acquisition. Although these leaves were kept fresh until 7 days AAP, TPP feeding might have been affected by the physiological changes in those leaves over time. Further investigation using whole plants for acquisition is required to confirm that. In studies carried out by Rashed et al. (2012), the highest percentage of acquisition success of Lso occurred when TPP had access to the whole plant and a higher proportion of TPP acquired Lso from stem tissue compared with leaflets and petioles. The effect of feeding site on acquisition success has been shown to be important in other vector-borne pathogen systems where the pathogen was heterogeneously distributed within the host (Daugherty et al. 2010).

Understanding the nature of the relationship between Lso and TPP is important for developing more effective control measures for zebra chip disease. Presumably a latent period is essential for Lso to multiply and enter the salivary gland of the psyllid before inoculation of another plant by the insect can occur. Munyaneza (2012) has reported that latent period in TPP is about 2 weeks after an acquisition period of 8-24 h on Lso-infected potato plants. He also stated that the latent period 
has been observed to be shorter when psyllids have fed on Lso-infected tomato plants, apparently because of a much higher Lso titre in tomato than potato. This study provides preliminary information on the time that might be required for Lso to multiply in the bodies of TPP.

\section{ACKNOWLEDGEMENTS}

We thank Robin Gardner-Gee and Joanne Poulton for their valuable comments on the earlier version of this paper, and Yilin Jia for help with statistics. We also thank Vicky Davis and Aleise Puketapu for TPP colony maintenance and Robin Gardner-Gee for regular screening of Lso-positive and -negative TPP colonies. This research was funded by The New Zealand Institute for Plant \& Food Research Limited.

\section{REFERENCES}

Alfaro-Fernandez A, Cebrian MC, Villaescusa FJ, Hermoso de Mendoza A, Ferrandiz JC, Sanjuan S, Font MI 2012a. First report of 'Candidatus Liberibacter solanacearum' in carrot in mainland Spain. Plant Disease 96: 582.

Alfaro-Fernandez A, Siverio F, Cebrian MC, Villaescusa FJ, Font MI 2012b. 'Candidatus Liberibacter solanacearum' associated with Bactericera trigonica-affected carrots in the Canary Islands. Plant Disease 96: 581.

Ammar E, Shatters RG, Jr., Hall DG 2011. Localization of Candidatus Liberibacter asiaticus, associated with citrus huanglongbing disease, in its psyllid vector using fluorescence in situ hybridization. Journal of Phytopathology 159: 726-734.

Beard SS, Pitman AR, Kraberger S, Scott IAW 2013. SYBR Green real-time quantitative PCR for the specific detection and quantification of 'Candidatus Liberibacter solanacearum' in field samples from New Zealand. European Journal of Plant Pathology 136(1): 203-215.

Beard SS, Scott IAW 2013. A rapid method for the detection and quantification of the vectorborne bacterium 'Candidatus Liberibacter solanacearum' in the tomato potato psyllid, Bactericera cockerelli. Entomologia Experimentalis et Applicata 147: 196-200
Buchman JL, Sengoda VG, Munyaneza JE 2011. Vector transmission efficiency of Liberibacter by Bactericera cockerelli (Hemiptera: Triozidae) in zebra chip potato disease: Effects of psyllid life stage and inoculation access period. Journal of Economic Entomology 104: 1486-1495.

Butler CD, Walker GP, Trumble JT 2012. Feeding disruption of potato psyllid, Bactericera cockerelli, by imidacloprid as measured by electrical penetration graphs. Entomologia Experimentalis et Applicata 142: 247-257.

Daugherty MP, Lopes JRS Almeida RPP 2010. Vector within-host preference mediates transmission of a heterogeneously distributed pathogen. Ecological Entomology 35: 360-366.

Hansen AK, Trumble JT, Stouthamer R, Paine TD 2008. A new huanglongbing species, "Candidatus liberibacter psyllaurous," found to infect tomato and potato, is vectored by the psyllid Bactericera cockerelli (Sulc). Applied and Environmental Microbiology 74: 5862-5865.

Inoue $\mathrm{H}$, Ohnishi J, Ito $\mathrm{T}$, Tomimura $\mathrm{K}$, Miyata S, Iwanami T, Ashihara W 2009. Enhanced proliferation and efficient transmission of Candidatus Liberibacter asiaticus by adult Diaphorina citri after acquisition feeding in the nymphal stage. Annals of Applied Biology 155: 29-36.

Levy J, Ravindran A, Gross D, Tamborindeguy C, Pierson E 2011. Translocation of 'Candidatus Liberibacter solanacearum', the zebra chip pathogen, in potato and tomato. Phytopathology 101: 1285-1291.

Liefting LW, Perez-Egusquiza ZC, Clover GRG, Anderson JAD 2008. A new 'Candidatus Liberibacter' species in Solanum tuberosum in New Zealand. Plant Disease 92: 1474.

Liefting LW, Sutherland PW, Ward LI, Paice KL, Weir BS, Clover RG 2009. A new 'Candidatus Liberibacter' species associated with diseases of Solanaceous crops. Plant Disease 93: 208-214. Martin NA 2008. Host plants of the potato/tomato psyllid: a cautionary tale. The Weta 35: 12-16.

Munyaneza JE 2012. Zebra chip disease of potato: biology, epidemiology, and management. American Journal of Potato Research 89: 329350 . 
Munyaneza JE, Crosslin JM, Upton JE 2007. Association of Bactericera cockerelli (Homoptera: Psyllidae) with "Zebra Chip," a new potato disease in Southwestern United States and Mexico. Journal of Economic Entomology 100: 656-663.

Ogden SC 2011. Tomato potato psyllid and liberibacter in New Zealand - impact and research programme overview. In: Workneh F, Rashed A, Rush CM ed. Proceedings of the 11th Annual Zebra Chip Reporting Session, San Antonio, Texas, USA, 6-9 November 2011. Pp. 173-177.

Pelz-Stelinski KS, Brlansky RH, Ebert TA, Rogers ME 2010. Transmission parameters for Candidatus Liberibacter asiaticus by Asian Citrus Psyllid (Hemiptera: Psyllidae). Journal of Economic Entomology 103: 1531-1541.

Puketapu AJ, Gardner-Gee R, Tooman LK, Beard SS 2013. A survey of Maori potato (taewa) seed tubers for the presence of Candidatus Liberibacter solanacearum. New Zealand Plant Protection 66: 356-364.

Purcell AH 1982. Insect vector relationships with prokaryotic plant pathogens. Annual Review of Phytopathology 20: 397-417.

Rashed AN TD, Paetzold L, Workneh F, Rush CM 2012. Transmission efficiency of 'Candidatus Liberibacter solanacearum' and potato zebra chip disease progress in relation to pathogen titer, vector numbers, and feeding sites. Phytopathology 102: 1079-1085.
Sandanayaka WRM, Page-Weir NEM, Fereres A 2011. Real time EPG recordings of tomatopotato psyllid (Bactericera cockerelli) feeding on tomato. New Zealand Plant Protection 64: 294 (abstract only).

Sandanayaka WRM, Moreno A, Tooman L, Page-Weir N, Fereres A 2012. Stylet penetration activities of tomato potato psyllid associated with acquisition and inoculation of Candidatus Liberibacter solanacearum. $24^{\text {th }}$ International Congress of Entomology, Daegu, Korea. CD abstract No. O401M07.

SAS Institute Inc. 2008. SAS/STAT 9.2, User's Guide, Cary, NC: SAS Institute Inc.

Tjallingii WF 1978. Electronic recording of penetration behaviour by aphids. Entomologia Experimentalis et Applicata 24: 721-730.

Tjallingii WF 1986. Wire effect on aphids during electrical recording of stylet penetration. Entomologia Experimentalis et Applicata 40: 89-98.

Teulon DAJ, Workman PJ, Thomas KL, Nielsen MC 2009. Bactericera cockerelli: incursion, dispersal and current distribution on vegetable crops in New Zealand. New Zealand Plant Protection 62: 136-144. 